\title{
An Evaluation Method for Sortie Generation Capacity of Carrier Aircrafts with Principal Component Reduction and Catastrophe Progression Method
}

\author{
Guoqing Xia, Tiantian Luan, and Mingxiao Sun \\ College of Automation, Harbin Engineering University, Harbin, China \\ Correspondence should be addressed to Tiantian Luan; luantiantian1988@126.com
}

Received 2 March 2017; Accepted 2 May 2017; Published 28 May 2017

Academic Editor: Ivan Giorgio

Copyright (c) 2017 Guoqing Xia et al. This is an open access article distributed under the Creative Commons Attribution License, which permits unrestricted use, distribution, and reproduction in any medium, provided the original work is properly cited.

\begin{abstract}
This study proposes a new comprehensive evaluation method with principal component reduction and catastrophe progression method, considering the complexity, hierarchy, contradiction, and relevance of the factors in sortie generation of carrier aircrafts. First of all, the index system of sortie generation capacity is ascertained, which contains correlative indexes. The principal component reduction method is applied to transform the correlative indexes into independent indexes. This method eliminates the effect of correlativity among indexes. The principal components are determined with their contributions. Then the reduced principal components are evaluated in catastrophe progression method. This method is easy to realize without weights, which is more objective. In fact, catastrophe progression method is a multidimensional fuzzy membership function, which is suitable for the incompatible multiobjective evaluation. Thus, a two-level evaluation method for sortie generation capacity of carrier aircrafts is realized with principal component reduction and catastrophe progression method. Finally, the Surge operation of aircraft carrier "Nimitz" is taken as an example to evaluate the sortie generation capacity. The results of the proposed method are compared with those of Analytic Hierarchy Process, which verify the usefulness and reliability of the proposed method.
\end{abstract}

\section{Introduction}

Aircraft carrier is the important part in the modern naval warfare. The research on the warfare capacity of aircraft carrier has become a hot issue with the increasing attention of the security in the territorial sea [1-3]. The comparison of sortie generation capacity of aircraft carrier in different operational schemes is helpful to determine the final plan [4-7]. Therefore, the evaluation for sortie generation capacity of aircraft carrier has important theoretical significance and application value.

The sortie generation capacity of aircraft carrier is mostly evaluated by Analytic Hierarchical Process (AHP) at present. The evaluation results are obtained by subjective scores of experts. Reference [8] studied the application of AHP in the measurement process. Reference [9] evaluated the original purchase process with AHP. Reference [10] developed an evaluation tool for the information sharing of supply chain using AHP. Reference [11] discussed the application of AHP in the process of risk assessment. An improved AHP in [12] was used in the priority scheduling problems. Reference [13] researched the application of AHP in business management. Reference [14] proposed the combination of fuzzy theory and AHP and discussed the consistency problem of the evaluation method. Reference [15] pointed out the shortcomings and improvements of AHP. Reference [16] studied the evaluation process of comprehensive method of fuzzy AHP. Reference [17] solved mining selection problem based on AHP and fuzzy mathematics. However, these evaluation methods are one-sided and subjective, which ignore the correlation and contradiction of indexes. AHP is difficult to evaluate the multivariate evaluation objects objectively [18-26].

A new evaluation method of principal component reduction (PCR) and catastrophe progression method (CPM) is proposed to evaluate the sortie generation capacity of carrier aircrafts in this study. The proposed method can avoid the subjectivity and complexity in the traditional evaluation method. The main contents are as follows. Firstly, the hierarchy structure of index system for sortie generation capacity is 


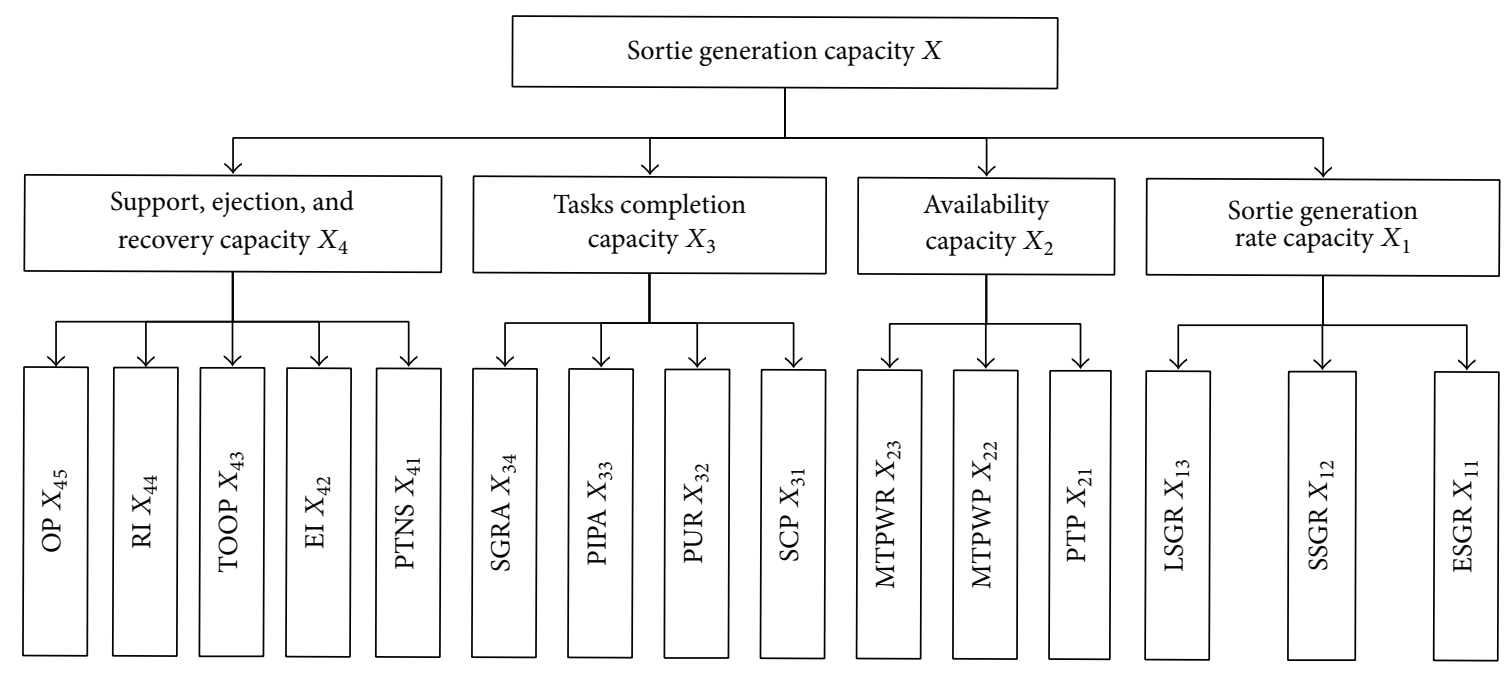

FIGURE 1: Index system for sortie generation of carrier aircrafts.

determined. Secondly, the related indexes are transformed to independent principal components by PCR. Then, independent principal components are evaluated by CPM. Finally, the usefulness and reliability of the new method are verified by comparing with the traditional evaluation method.

\section{Index System for Sortie Generation Capacity of Carrier Aircrafts}

The index system for sortie generation capacity of carrier aircrafts is established with related research results. A threelevel index system with complexity, hierarchy, contradiction, and relevance is established by recursive hierarchy method. The index system for sortie generation capacity of carrier aircrafts is shown in Figure 1.

These indexes are defined as follows.

(1) Emergency sortie generation rate (ESGR): it is the maximum number of ready aircrafts taking off in a few minutes.

(2) Surge sortie generation rate (SSGR): it is the average number of aircrafts per day in the Surge operation (4 days).

(3) Last sortie generation rate (LSGR): it is the average number of aircrafts per day in the continuous operation (30 days).

(4) Performing tasks proportion (PTP): it is the time proportion that the aircrafts can carry out one task at least under a certain flight plan and logistics condition.

(5) Missing tasks proportion waiting for parts (MTPWP): it is the proportion of aircrafts missing the tasks due to waiting for parts.

(6) Missing tasks proportion waiting for repair (MTPWR): it is the proportion of aircrafts missing the tasks due to waiting for repair.

(7) Scheduled completion proportion (SCP): it is the proportion of completed number in the planned number of aircrafts.

(8) Pilot utilization rate (PUR): it is the average utilization rate of the pilots per day.
(9) Plan implementation probability per aircraft (PIPA): it is the plan implementation probability per aircraft under the certain constraints in a given period of time.

(10) Sortie generation rate per aircraft (SGRA): it is the sortie generation rate per aircraft under the certain constraints.

(11) Preparation time for next sortie (PTNS): it is the preparation time for next sortie under the condition of a certain resource allocation.

(12) Ejection interval (EI): it is the average time for ejecting a single aircraft per catapult.

(13) Take-off outage proportion (TOOP): it is the proportion of canceled number in the ready number of aircrafts.

(14) Recovery interval (RI): it is the average time for recovering a single aircraft.

(15) Overshoot proportion (OP): it is the proportion of number of aircrafts failed to recover in the number of aircrafts ready to recover.

\section{Principal Component Reduction Method}

3.1. Principal Component Reduction Principle. There are correlations between various indexes for sortie generation capacity of carrier aircrafts, which will bring repetitive information. The independent indexes can be obtained from related indexes using principal component reduction method. This method can minimize the information loss after reduction.

Principal component reduction uses dimension reduction techniques to obtain less comprehensive variables instead of the original variables. These comprehensive variables cover the most information of the original variables. Then the objective phenomenon is evaluated by calculating the score of comprehensive principal component.

3.2. Steps of Principal Component Reduction. Steps of principal component reduction are as shown in Figure 2. 


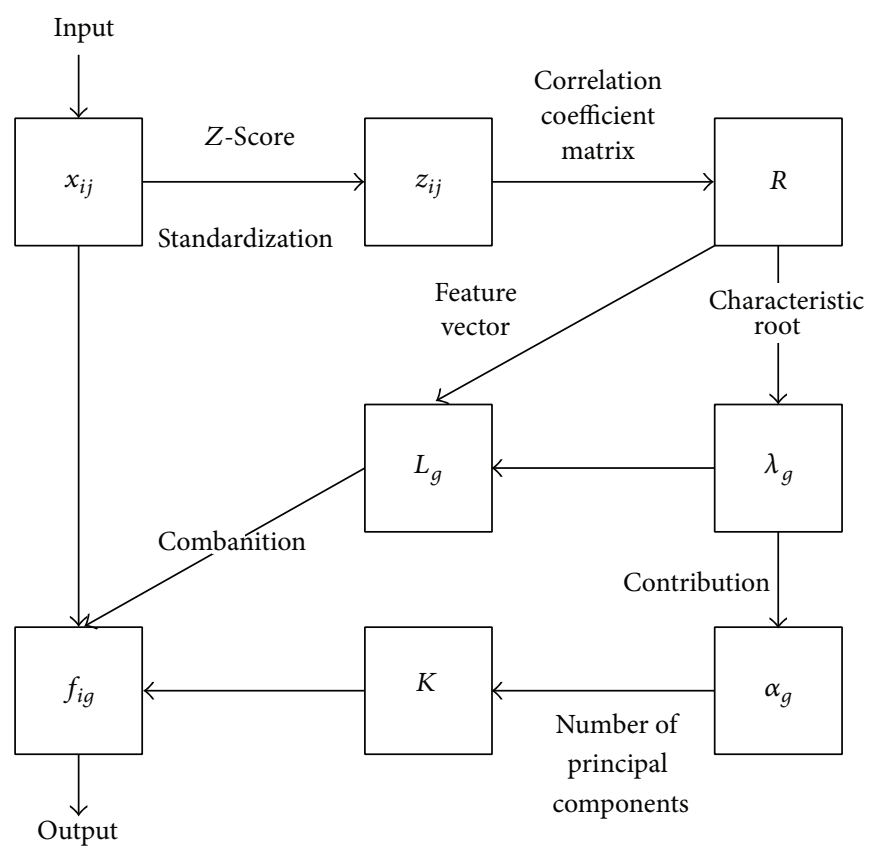

FIGURE 2: Steps of principal component reduction.

Specific steps are as follows.

Step 1 (parameters standardization). Each index is nondimensionalized due to the different dimensions of indexes. The numerical transformation can eliminate the dimensional effect of indexes. $Z$-Score method is applied to transform the original matrix $X=\left[x_{i j}\right]_{n \times m}$ to standardized matrix $Z=\left[z_{i j}\right]_{n \times m}$, where $n$ is the number of scenarios and $m$ is the number of indexes:

$$
z_{i j}=\frac{x_{i j}-\overline{x_{j}}}{s_{j}},
$$

where $\overline{x_{j}}$ is the mean of $j$ th index and $s_{j}$ is the standard deviation of $j$ th index.

Step 2 (correlation coefficient matrix $R$ ).

$$
r_{j k}=\frac{1}{n-1} \sum_{i=1}^{n} z_{i j} z_{i k},
$$

where $R=\left[r_{j k}\right]_{m \times m}, r_{i i}=1$, and $r_{j k}=r_{k j}$.

Step 3 (characteristic roots of $R$ ). The characteristic roots of $R$ can be calculated by

$$
\left|\lambda_{g} I_{m}-R\right|=0
$$

where $\lambda_{g}(g=1,2, \ldots, m)$ is the characteristic root, which is the variance of principal component. It denotes the effect of each principal component on the evaluated object.

Step 4 (feature vectors of $R$ ). The feature vectors of $R$ can be obtained from

$$
\left[\lambda_{g} I_{m}-R\right] L_{g}=0
$$

where $L$ is a real-valued vector of $m$ dimensions. $L_{g}$ is the feature vector of $\lambda_{g}$ and the coefficient of $z_{j}$ in the new coordinate system.

Step 5 (contribution of $R$ ). $\alpha_{g}$ is the information amount of each component in the total information amount, which is the contribution

$$
\alpha_{g}=\frac{\lambda_{g}}{\sum_{g=1}^{m} \lambda_{g}} .
$$

Step 6 (number of principal components $K$ ). If the number of original variables is more, the first $K$ principal components are analyzed instead of the original variables and the other variables are ignored. The proportion of the $K$ principal components in the original variable information is $\alpha(K)$ :

$$
\alpha(K)=\left(\sum_{g=1}^{K} \lambda_{g}\right)\left(\sum_{g=1}^{m} \lambda_{g}\right)^{-1} .
$$

Thus, the number of principal components is determined with a balance between $K$ and $\alpha(K)$. On the one hand, the smaller $K$ is better. On the other hand, the larger $\alpha(K)$ is better. It will keep enough information with few components in this way. In this study, $\alpha(K) \geq 95 \%$.

Finally, $m$ related indexes can be transformed to $K$ independent principal components $f_{i 1}-f_{i K}$ :

$$
f_{i g}=\sum_{j=1}^{m} L_{i j} z_{i j} .
$$

The index system after reduction is shown in Figure 3. 


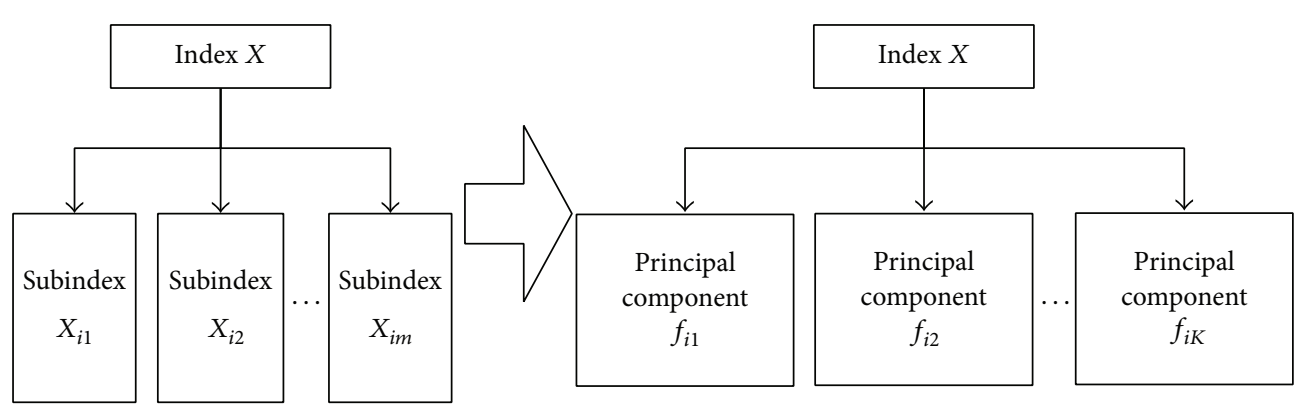

Figure 3: Index system after reduction.

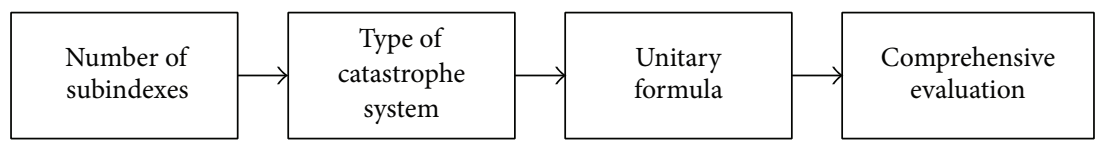

FIGURE 4: Steps of catastrophe progression method.

\section{Catastrophe Progression Evaluation Method}

4.1. Description of Catastrophe Progression Method. The index system for sortie generation capacity of carrier aircrafts after reduction is applied to evaluate. The contradiction decomposition of evaluated objects is the first step of catastrophe progression method. Then catastrophe fuzzy membership function is the combination of catastrophe theory and fuzzy mathematics. This method considers the relative importance of evaluation indexes instead of index weight, which reduces the subjectivity and simplifies calculation.

In the process of formulating combat scenario, a variety of scenarios are designed due to the influence of various factors. The scenarios are evaluated comprehensively in the process of selecting the optimal scenario. The evaluation process is conducted from the indexes in lower levels to the indexes in upper levels according to catastrophe progression method. Finally, a catastrophe progression between 0 and 1 can be obtained. If the catastrophe progression is bigger, the scenario is better.

4.2. Steps of Catastrophe Progression Method. The steps of catastrophe progression method are as shown in Figure 4.

Step 1 (type of catastrophe system). The type of catastrophe system is determined by the number of subindexes, which is shown in Table 1.

In Table $1, f(x)$ is the potential function of $x . a, b, c$, and $d$ are subindexes, which are sorted from high importance to low importance.

Step 2 (unitary formula). The critical points of potential function $f(x)$ gather to a balance surface based on catastrophe theory, which can be obtained from the first-order derivative of $f(x)$ :

$$
f^{\prime}(x)=0
$$

TABLE 1: Type of catastrophe system.

\begin{tabular}{lcc}
\hline Type & $\begin{array}{c}\text { Number of } \\
\text { subindexes }\end{array}$ & System model \\
\hline $\begin{array}{l}\text { Sharp point } \\
\text { type }\end{array}$ & 2 & $f(x)=x^{4}+a x^{2}+b x$ \\
Dovetail type & 3 & $\frac{1}{5} x^{5}+\frac{1}{3} a x^{3}+\frac{1}{2} b x^{2}+c x$ \\
& & $f(x)=\frac{1}{6} x^{6}+\frac{1}{4} a x^{4}+$ \\
Butterfly type & 4 & $\frac{1}{3} b x^{3}+\frac{1}{2} c x^{2}+d x$ \\
\hline
\end{tabular}

The singular points of potential function $f(x)$ can be obtained by the second-order derivative:

$$
f^{\prime \prime}(x)=0 \text {. }
$$

The unitary formula can be derived from bifurcation set, which will transform different states of subindex to the same state.

(1) Bifurcation set equations of sharp point system are

$$
\begin{aligned}
& a=-6 x^{2}, \\
& b=8 x^{3} .
\end{aligned}
$$

Then the normalization formula can be derived from

$$
\begin{aligned}
& x_{a}=a^{1 / 2}, \\
& x_{b}=b^{1 / 3},
\end{aligned}
$$

where $x_{a}$ is the value of $x$ corresponding $a . x_{b}$ is the value of $x$ corresponding $b$.

(2) Bifurcation set equations of dovetail system are

$$
\begin{aligned}
& a=-6 x^{2}, \\
& b=8 x^{3}, \\
& c=-3 x^{4} .
\end{aligned}
$$


Then the normalization formula can be derived from

$$
\begin{aligned}
& x_{a}=a^{1 / 2}, \\
& x_{b}=b^{1 / 3}, \\
& x_{c}=c^{1 / 4} .
\end{aligned}
$$

(3) Bifurcation set equations of butterfly system are

$$
\begin{aligned}
& a=-10 x^{2}, \\
& b=20 x^{3}, \\
& c=-15 x^{4}, \\
& d=4 x^{5} .
\end{aligned}
$$

Then the normalization formula can be derived from

$$
\begin{aligned}
& x_{a}=a^{1 / 2}, \\
& x_{b}=b^{1 / 3}, \\
& x_{c}=c^{1 / 4}, \\
& x_{d}=d^{1 / 5} .
\end{aligned}
$$

Normalization formula is a multidimensional fuzzy membership function.

Step 3 (comprehensive evaluation with normalization formula). The ideal strategy is obtained from (16), when the fuzzy targets are $A_{1}, A_{2}, \ldots, A_{m}$ in the same scenario

$$
C=A_{1} \cap A_{2} \cap \cdots \cap A_{m} .
$$

The membership function is

$$
\mu(x)=\mu_{A_{1}}(x) \wedge \mu_{A_{2}}(x) \wedge \cdots \wedge \mu_{A_{m}}(x),
$$

where $\mu_{A_{i}}(x)$ is the membership function of $A_{i}$. If the indexes are complementary, the membership function is the average value of $\mu_{A_{i}}(x)$.

\section{Evaluation for Sortie Generation Capacity of Carrier Aircrafts}

5.1. Evaluation Samples. The object of evaluation is the Surge operation of "Nimitz" carrier in 1997. Ten scenarios are selected randomly in order to ensure the scientific nature, which are shown in Tables 2-5. In Tables $2-5, X_{11}$ is emergency sortie generation rate, $X_{12}$ is surge sortie generation rate, $X_{13}$ is last sortie generation rate, $X_{21}$ is performing tasks proportion, $X_{22}$ is missing tasks proportion waiting for parts, $X_{23}$ is missing tasks proportion waiting for repair, $X_{31}$ is scheduled completion proportion, $X_{32}$ is pilot utilization rate, $X_{33}$ is plan implementation probability per aircraft, $X_{34}$ is sortie generation rate per aircraft, $X_{41}$ is preparation time for next sortie, $X_{42}$ is ejection interval, $X_{43}$ is take-off outage proportion, $X_{44}$ is recovery interval, and $X_{45}$ is overshoot proportion. The data in Tables $2-5$ are the original data.
TABLE 2: Index $X_{1}$ of sortie generation rate capacity.

\begin{tabular}{lccc}
\hline Scenario & $X_{11}$ (sortie) & $X_{12}$ (sortie/day) & $X_{13}$ (sortie/day) \\
\hline 1 & 30 & 250 & 200 \\
2 & 31 & 240 & 180 \\
3 & 29 & 235 & 210 \\
4 & 33 & 260 & 220 \\
5 & 32 & 210 & 170 \\
6 & 29 & 245 & 194 \\
7 & 27 & 267 & 230 \\
8 & 32 & 211 & 183 \\
9 & 25 & 261 & 201 \\
10 & 32 & 232 & 196 \\
\hline
\end{tabular}

TABLE 3: Index $X_{2}$ of aircraft availability capacity.

\begin{tabular}{lccc}
\hline Scenario & $X_{21}(\%)$ & $X_{22}(\%)$ & $X_{23}(\%)$ \\
\hline 1 & 80 & 11 & 9 \\
2 & 85 & 20 & 5 \\
3 & 90 & 4 & 6 \\
4 & 75 & 11 & 14 \\
5 & 82 & 10 & 18 \\
6 & 91 & 3 & 5 \\
7 & 78 & 23 & 9 \\
8 & 84 & 9 & 7 \\
9 & 85 & 1 & 4 \\
10 & 72 & 2 & 16 \\
\hline
\end{tabular}

TABLE 4: Index $X_{3}$ of tasks completion capacity.

\begin{tabular}{lcccc}
\hline Scenario & $X_{31}(\%)$ & $X_{32}$ (sortie/day) & $X_{33}(\%)$ & $X_{34}$ (sortie/day) \\
\hline 1 & 85 & 2.5 & 90 & 6 \\
2 & 74 & 2.2 & 80 & 7 \\
3 & 81 & 2.0 & 84 & 5 \\
4 & 90 & 1.8 & 75 & 6 \\
5 & 61 & 1.5 & 68 & 5 \\
6 & 86 & 1.9 & 86 & 8 \\
7 & 78 & 2.1 & 88 & 5 \\
8 & 65 & 2.3 & 94 & 6 \\
9 & 79 & 2.4 & 81 & 7 \\
10 & 83 & 1.7 & 82 & 5 \\
\hline
\end{tabular}

5.2. Indexes Reduction. Take the reduction process of index $\mathrm{X}_{4}$ as an example.

Step 1 (standardization). The Z-Score method is used to standardize indexes, and the results are as shown in Table 6. In Table $6, Z_{41}, Z_{42}, Z_{43}$, and $Z_{44}$ are standardize indexes. 
TABLE 5: Index $X_{4}$ of support, ejection, and recovery capability.

\begin{tabular}{lccccc}
\hline Scenario & $\begin{array}{c}X_{41} \\
\text { (minute) }\end{array}$ & $\begin{array}{c}X_{42} \\
\text { (minute) }\end{array}$ & $X_{43}(\%)$ & $\begin{array}{c}X_{44} \\
\text { (minute) }\end{array}$ & $\begin{array}{c}X_{45} \\
(\%)\end{array}$ \\
\hline 1 & 30 & 1 & 1 & 1.5 & 3.3 \\
2 & 32 & 2 & 0.5 & 1.8 & 5 \\
3 & 28 & 1.4 & 1.2 & 1.4 & 1 \\
4 & 25 & 1.6 & 1.6 & 1.9 & 7 \\
5 & 33 & 2.5 & 2 & 2.2 & 6 \\
6 & 45 & 1.1 & 3 & 1.1 & 2 \\
7 & 27 & 0.6 & 0.6 & 1.2 & 10 \\
8 & 26 & 0.7 & 0.8 & 1.6 & 3 \\
9 & 36 & 0.5 & 1.6 & 1.7 & 4.5 \\
10 & 29 & 1.2 & 0.4 & 2.1 & 6 \\
\hline
\end{tabular}

TABLE 6: Standardization of index $X_{4}$.

\begin{tabular}{lccccc}
\hline Scenario & $Z_{41}$ & $Z_{42}$ & $Z_{43}$ & $Z_{44}$ & $Z_{45}$ \\
\hline 1 & -0.1854 & -0.4086 & -0.3352 & -0.4134 & -0.5609 \\
2 & 0.1517 & 1.1630 & -0.9558 & 0.4134 & 0.0834 \\
3 & -0.5224 & 0.2200 & -0.0869 & -0.6890 & -1.4326 \\
4 & -1.0280 & 0.5343 & 0.4096 & 0.6890 & 0.8414 \\
5 & 0.3202 & 1.9487 & 0.9062 & 1.5157 & 0.4624 \\
6 & 2.3425 & -0.2515 & 2.1475 & -1.5157 & -1.0536 \\
7 & -0.6909 & -1.0372 & -0.8317 & -1.2402 & 1.9784 \\
8 & -0.8595 & -0.8801 & -0.5834 & -0.1378 & -0.6746 \\
9 & 0.8258 & -1.1944 & 0.4096 & 0.1378 & -0.1061 \\
10 & -0.3539 & -0.0943 & -1.0799 & 1.2402 & 0.4624 \\
\hline
\end{tabular}

Step 2 (correlation coefficient matrix). One has

$R$

$$
=\left[\begin{array}{ccccc}
1 & 0.0159 & 0.7398 & -0.2916 & -0.3731 \\
0.0159 & 1 & 0.1600 & 0.5919 & 0.0279 \\
0.7398 & 0.1600 & 1 & -0.2186 & -0.3401 \\
-0.2916 & 0.5919 & -0.2186 & 1 & 0.2588 \\
-0.3731 & 0.0279 & -0.3401 & 0.2588 & 1
\end{array}\right] .
$$

Step 3 (characteristic roots). One has

$$
\begin{aligned}
& {\left[\begin{array}{lllll}
\lambda_{1} & \lambda_{2} & \lambda_{3} & \lambda_{4} & \lambda_{5}
\end{array}\right]} \\
& \quad=\left[\begin{array}{llllll}
2.1781 & 1.5206 & 0.7310 & 0.2381 & 0.3322
\end{array}\right] .
\end{aligned}
$$

Step 4 (feature vectors). One has

$$
\begin{aligned}
& {\left[\begin{array}{llllll}
L_{1} & L_{2} & L_{3} & L_{4} & L_{5}
\end{array}\right]} \\
& =\left[\begin{array}{ccccc}
-0.5778 & -0.2117 & -0.2907 & -0.6141 & -0.3996 \\
0.1322 & -0.7342 & 0.0912 & -0.2976 & 0.5888 \\
-0.5450 & -0.3264 & -0.3113 & 0.6972 & 0.1161 \\
0.4056 & -0.5542 & 0.0927 & 0.2136 & -0.6886 \\
0.4326 & 0.0500 & -0.8954 & -0.0512 & 0.0780
\end{array}\right] .
\end{aligned}
$$

Step 5 (contribution). One has

$$
\begin{aligned}
& {\left[\begin{array}{lllll}
\alpha_{1} & \alpha_{2} & \alpha_{3} & \alpha_{4} & \alpha_{5}
\end{array}\right]} \\
& \quad=\left[\begin{array}{llllll}
0.4356 & 0.3041 & 0.1462 & 0.0476 & 0.0664
\end{array}\right]
\end{aligned}
$$

Step 6 (number of principal components). Let $\alpha(K) \geq 95 \%$; then sort $\alpha$ from big to small:

$$
\begin{aligned}
& {\left[\begin{array}{lllll}
\alpha_{1} & \alpha_{2} & \alpha_{3} & \alpha_{5} & \alpha_{4}
\end{array}\right]} \\
& \quad=\left[\begin{array}{llllll}
0.4356 & 0.3041 & 0.1462 & 0.0664 & 0.0476
\end{array}\right]
\end{aligned}
$$

When $K=4, \alpha(K)=95.24 \% \geq 95 \%$.

Step 7 (principal components). The principal components of index $X_{4}$ are $f_{41}, f_{42}, f_{43}$, and $f_{44}$, which are determined by characteristic roots, feature vectors, and the number of principal components:

$$
\begin{aligned}
f_{41}= & -0.3915 X_{41}+0.1072 X_{42}-0.6375 X_{43} \\
& +0.8312 X_{44}+0.7505 X_{45}, \\
f_{42}= & -0.1434 X_{41}-0.5954 X_{42}-0.3818 X_{43} \\
& -1.1359 X_{44}+0.0868 X_{45}, \\
f_{43}= & -0.1970 X_{41}+0.0740 X_{42}-0.3641 X_{43} \\
& +0.1900 X_{44}-1.5535 X_{45}, \\
f_{44}= & -0.2708 X_{41}+0.4774 X_{42}+0.1357 X_{43} \\
& -1.4112 X_{44}+0.1354 X_{45} .
\end{aligned}
$$

Similarly, the principal components of index $X_{1}, X_{2}$, and $X_{3}$ can be derived by repeating Steps $1-7$ :

$$
\begin{aligned}
f_{11}= & -1.2109 X_{11}+0.7943 X_{12}+0.3977 X_{13}, \\
f_{12}= & 2.0214 X_{11}+0.2061 X_{12}+0.3569 X_{13}, \\
f_{13}= & 0.5796 X_{11}+0.9406 X_{12}-0.4140 X_{13}, \\
f_{21}= & 0.5329 X_{21}-0.4290 X_{22}-0.6711 X_{23}, \\
f_{22}= & -0.0095 X_{21}-1.8995 X_{22}+0.2937 X_{23}, \\
f_{23}= & -0.5352 X_{21}-0.3935 X_{22}-0.6795 X_{23}, \\
f_{31}= & 0.5573 X_{31}+0.7273 X_{32}+0.5758 X_{33} \\
& +0.3004 X_{34}, \\
f_{32}= & 1.4342 X_{31}-0.3351 X_{32}-0.3749 X_{33} \\
& +0.3459 X_{34}, \\
f_{33}= & 1.1895 X_{31}-0.0913 X_{32}+0.3033 X_{33} \\
& -0.5228 X_{34}, \\
f_{34}= & 0.2282 X_{31}+0.8156 X_{32}-0.6306 X_{33} \\
& -0.1828 X_{34} \cdot
\end{aligned}
$$




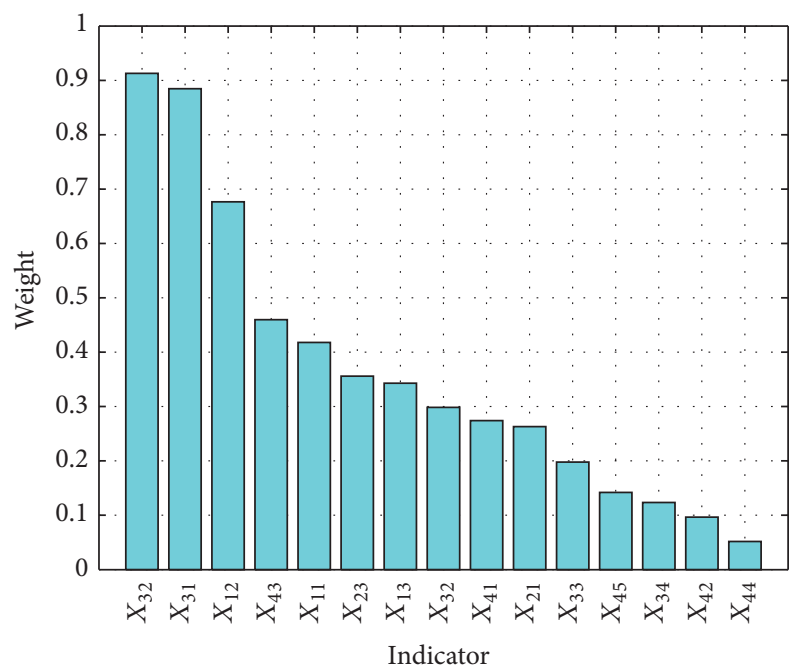

FIGURE 5: Weights of sortie generation capacity indexes.

The comprehensive scoring model can be obtained from (23) and (24) and the contributions:

$$
\begin{aligned}
Y_{1}= & 0.4180 X_{11}+0.6767 X_{12}+0.3430 X_{13}, \\
Y_{2}= & 0.2630 X_{21}-0.9130 X_{22}-0.3558 X_{23}, \\
Y_{3}= & 0.8846 X_{31}+0.2985 X_{32}+0.1979 X_{33} \\
& +0.1235 X_{34}, \\
Y_{4}= & -0.2740 X_{41}-0.0964 X_{42}-0.4599 X_{43} \\
& -0.0518 X_{44}-0.1420 X_{45} .
\end{aligned}
$$

The weights of indexes are shown in Figure 5 according to (25). In Figure 5, the horizontal axis is the evaluated index and the vertical axis is the weight of index.

Figure 5 shows that the most important subindexes are the pilot utilization rate and scheduled completion proportion, the weights of which are greater than weights of other indexes.

5.3. Catastrophe Progression Evaluation. The index system after reduction is shown in Figure 6. follows.

The steps of catastrophe progression evaluation are as

Step 1 (normalization). Take the principal components $f_{11}$, $f_{12}$, and $f_{13}$ of index $X_{1}$ as the example. The results are shown in Table 7. In Table 7, $f_{11}, f_{12}$, and $f_{13}$ are normalization of principal components.

Step 2 (calculate evaluation value). The evaluation values of catastrophe progression for indexes $X_{1}, X_{2}, X_{3}$, and $X_{4}$ are calculated. The number of subindexes for indexes $X_{1}$ and $X_{2}$ is three; then the type of catastrophe is dovetail type. The number of subindexes for indexes $X_{3}$ and $X_{4}$ is four; then the type of catastrophe is butterfly type. Thus the evaluation values are shown in Table 8 . In Table $8, X_{1}$ is sortie generation
TABLE 7: Normalization of principal components.

\begin{tabular}{lccc}
\hline Scenario & $f_{11}$ & $f_{12}$ & $f_{13}$ \\
\hline 1 & 0.5301 & 0.5716 & 0.6225 \\
2 & 0.3134 & 0.6165 & 0.7314 \\
3 & 0.5451 & 0.4590 & 0 \\
4 & 0.4320 & 1 & 1 \\
5 & 0 & 0.6593 & 0.2127 \\
6 & 0.5541 & 0.4282 & 0.4463 \\
7 & 0.9863 & 0.3299 & 0.3273 \\
8 & 0.0532 & 0.6986 & 0.0815 \\
9 & 1 & 0 & 0.2813 \\
10 & 0.2390 & 0.7688 & 0.4574 \\
\hline
\end{tabular}

TABLE 8: Evaluation values of indexes $X_{1}, X_{2}, X_{3}$, and $X_{4}$.

\begin{tabular}{lcccc}
\hline Scenario & $X_{1}$ & $X_{2}$ & $X_{3}$ & $X_{4}$ \\
\hline 1 & 0.8154 & 0.7687 & 0.9483 & 0.8721 \\
2 & 0.7786 & 0.6630 & 0.8140 & 0.8525 \\
3 & 0.5032 & 0.9201 & 0.8629 & 0.8776 \\
4 & 0.8858 & 0.5842 & 0.9191 & 0.8212 \\
5 & 0.5165 & 0.3618 & 0.3380 & 0.6127 \\
6 & 0.7718 & 0.9456 & 0.6934 & 0.6853 \\
7 & 0.8135 & 0.3654 & 0.8388 & 0.7291 \\
8 & 0.5508 & 0.8386 & 0.5526 & 0.8818 \\
9 & 0.5761 & 0.9757 & 0.8906 & 0.8003 \\
10 & 0.7425 & 0.6427 & 0.8199 & 0.6519 \\
\hline
\end{tabular}

rate capacity, $X_{2}$ is availability capacity, $X_{3}$ is tasks completion capacity, and $X_{4}$ is support, ejection, and recovery capacity. The data in Table 8 are the evaluation values of the above four indexes.

Step 3 (calculate evaluation value of $X$ ). The number of subindexes for index $X$ is four; then the type of catastrophe is butterfly type. The evaluation results of 10 scenarios are shown in Table 9. $X$ is sortie generation capacity. The data in Table 9 are the evaluation values of sortie generation capacity.

5.4. Analysis of Evaluation Results. The evaluation results of the proposed method are compared with that of AHP, in order to verify the usefulness of the proposed method. The comparison is shown in Figure 7 and the deviations of evaluation results are shown in Figure 8. In Figure 7, the horizontal axis is the evaluated scenario and the vertical axis is the evaluation value. In Figure 8, the horizontal axis is the evaluated scenario and the vertical axis is the deviation of proposed method and AHP.

Figure 7 shows that that evaluation results of two methods are similar. Figure 8 shows the deviations of evaluation results, and the maximum absolute value of deviation is less than 0.05 , which verifies usefulness and reliability of the principal component reduction and catastrophe progression evaluation method. The proposed method can evaluate scenarios more objectively. 
TABLE 9: Evaluation values of index $X$.

\begin{tabular}{lcccccccccc}
\hline Scenario & 1 & 2 & 3 & 4 & 5 & 6 & 7 & 8 & 9 & 10 \\
\hline$X$ & 0.9663 & 0.9554 & 0.7237 & 0.9719 & 0.6233 & 0.9268 & 0.9239 & 0.8832 & 0.9453 & 0.9380 \\
\hline
\end{tabular}

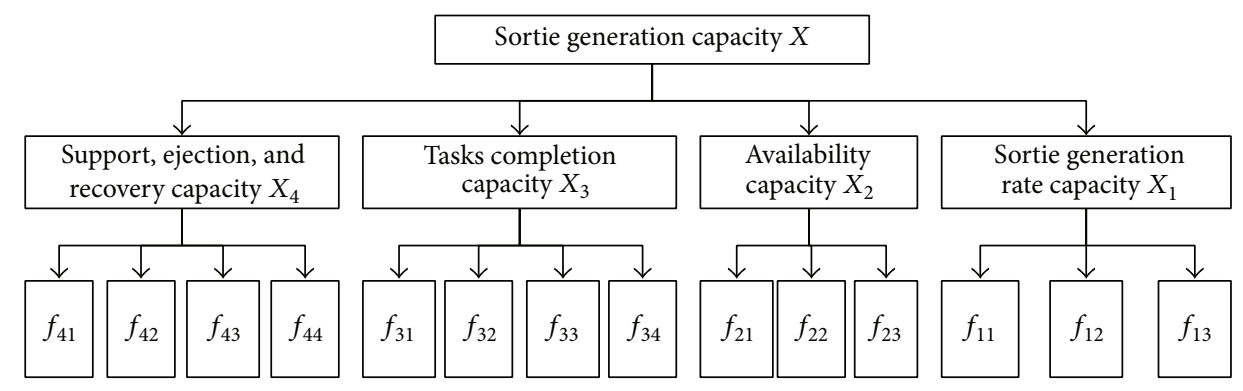

FIGURE 6: Index system after reduction.

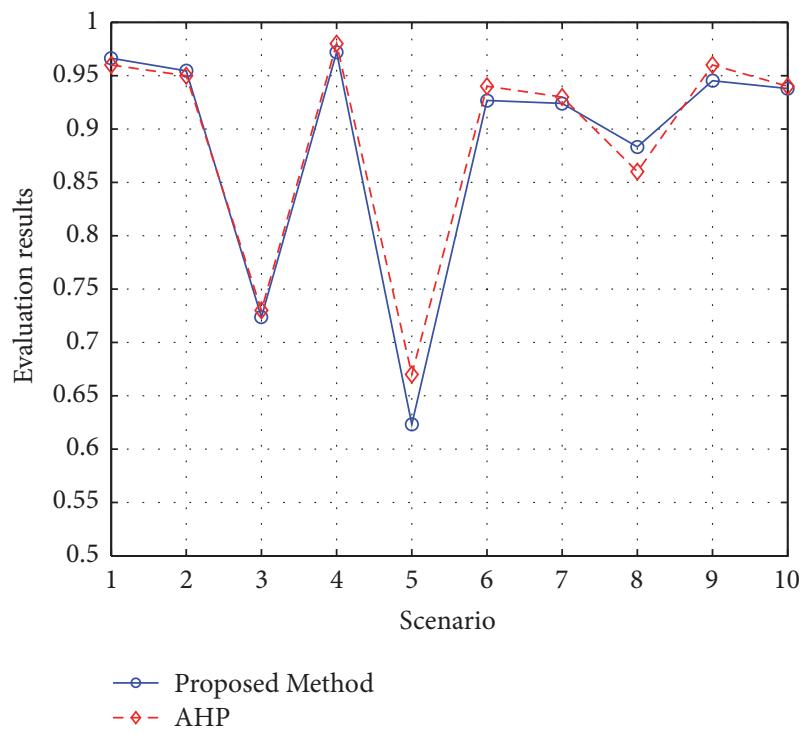

FIGURE 7: Comparison of evaluation results.

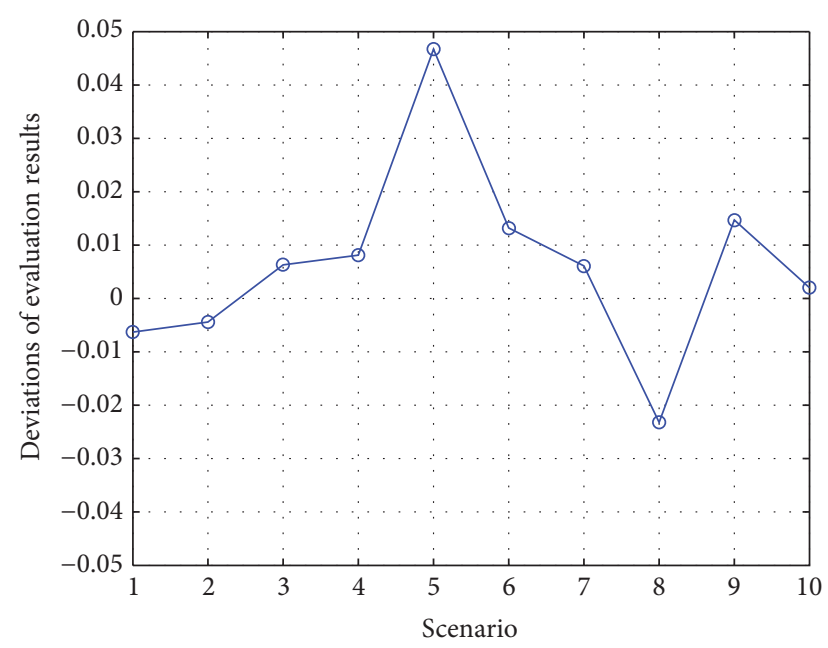

FIGURE 8: Deviations of evaluation results.

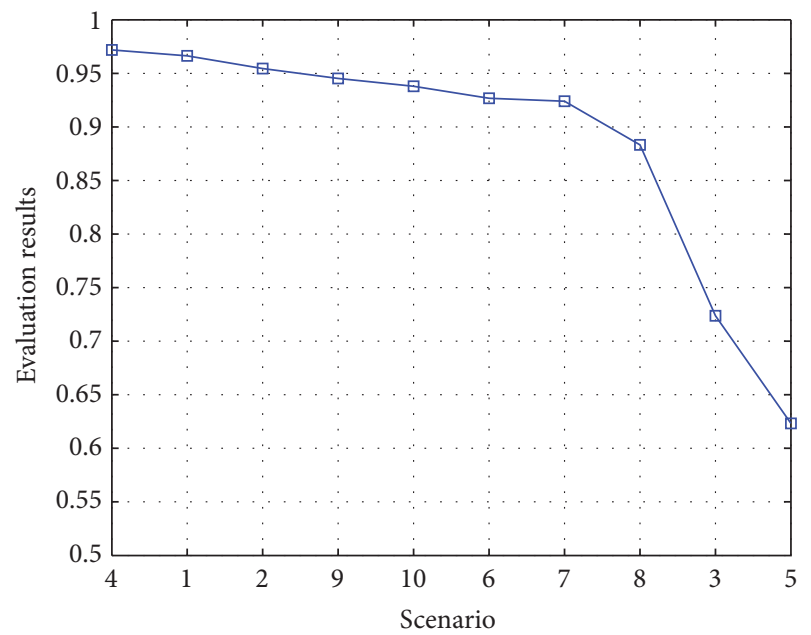

FIGURE 9: Order of scenarios index.

Therefore, the selected 10 scenarios can be sorted according to the comprehensive evaluation results, which is shown in Figure 9. In Figure 9, the horizontal axis is the evaluated scenario and the vertical axis is the evaluation value.

The Figure 9 shows that the best scenario is scenario 4 . Its comprehensive evaluation value is 0.9719 . And the worst scenario is scenario 5. Its comprehensive evaluation value is 0.6233 . The best scenario can be elected from the selected scenarios. Thus, the evaluation method will help decisionmaker to draw up a plan.

Therefore, the principal component reduction and catastrophe progression evaluation method can analyze the importance of indexes for sortie generation capacity and sort the selected scenarios objectively and reliably.

\section{Conclusions}

This study proposes a new comprehensive evaluation method based on principal component reduction and catastrophe progression method. First of all, the index system of sortie generation capacity is ascertained in Figure 1 and Tables 2-5, 
which contains correlative indexes. The principal component reduction method is applied to transform the correlative indexes into independent indexes in Figures 2, 5, and 6 and Table 6. This method eliminates the effect of correlativity among indexes. The principal components are determined with their contributions. Then the reduced principal components are evaluated in catastrophe progression method in Figures 3 and 4 and Table 1 . This method is easy to realize without weights, which is more objective. In fact, catastrophe progression method is a multidimensional fuzzy membership function, which is suitable for the incompatible multiobjective evaluation. Thus, a two-level evaluation method for sortie generation capacity of carrier aircrafts is realized with principal component reduction and catastrophe progression method. The principal component reduction and catastrophe progression evaluation method can analyze the importance of indexes for sortie generation capacity and sort the selected scenarios objectively and reliably in Figures 7-9 and Tables 7-9. At the same time, the proposed method is suitable for other evaluated objects.

\section{Nomenclature}

$X_{1}$ : Sortie generation rate capacity

$X_{2}$ : Availability capacity

$X_{3}$ : Tasks completion capacity

$X_{4}$ : Support, ejection, and recovery capacity

$X_{11}$ : Emergency sortie generation rate

$X_{12}$ : Surge sortie generation rate

$X_{13}$ : Last sortie generation rate

$X_{21}$ : Performing tasks proportion

$X_{22}$ : Missing tasks proportion waiting for parts

$X_{23}$ : Missing tasks proportion waiting for repair

$X_{31}$ : Scheduled completion proportion

$X_{32}$ : Pilot utilization rate

$X_{33}$ : Plan implementation probability per aircraft

$X_{34}$ : Sortie generation rate per aircraft

$X_{41}$ : Preparation time for next sortie

$X_{42}$ : Ejection interval

$X_{43}$ : Take-off outage proportion

$X_{44}$ : Recovery interval

$X_{45}$ : Overshoot proportion

$X$ : Original input matrix

$Z$ : $\quad$ Standardized matrix

$n$ : Number of scenarios

$m$ : Number of indexes

$\overline{x_{j}}:$ Mean of $j$ th index

$s_{j}$ : Standard deviation of $j$ th index

$R$ : Correlation coefficient matrix

$\lambda_{g}:$ Characteristic root

$L: \quad$ Real-valued vector

$L_{g}:$ Feature vector

$\alpha_{g}$ : Information amount of each component in the total information amount

$K$ : Number of principal components
$\alpha(K): \quad \quad \quad \quad$ Proportion of the $K$ principal components in the original variable information

$f_{i g}: \quad$ Independent principal components

$f(x)$ : Potential function

$a, b, c, d: \quad$ Subindex

$A_{1}, A_{2}, \ldots, A_{m}$ : Fuzzy targets

$\mu_{A_{i}}(x)$ : Membership function.

\section{Conflicts of Interest}

The authors declare that there are no conflicts of interest regarding the publication of this study.

\section{References}

[1] A. Jewell, M. A. Wigge, and C. M. K. Gagnon, "USS Nimitz and carrier airwing nine Surge demonstration," Alexandria: Center for Naval Analyses, 1988.

[2] G. Q. Xia, H. Z. Chen, and T. T. Luan, "Performance evaluating model for two echelon maintenance and support system under limited repair capacity," Systems Engineering-Theory and Practice, vol. 35, no. 4, pp. 1041-1047, 2015.

[3] D. P. Bian, T. T. Luan, and Y. Song, "A layout method of carrierbased aircraft based on simulated annealing," Applied Science and Technology, vol. 42, pp. 20-24, 2015.

[4] D. P. Bian, T. T. Luan, and Q. C. Mi, "Research on the problem of gate assignment for carrier aircraft deck," Measurement and Control Technology, vol. 33, pp. 150-153, 2014.

[5] G. Xia and T. Luan, "Study of ship heading control using RBF neural network," International Journal of Control and Automation, vol. 8, no. 10, pp. 227-236, 2015.

[6] L. Liang, M. Sun, S. Zhang, Y. Wen, P. Zhao, and J. Yuan, "Control system design of anti-rolling tank swing bench using BP neural network PID based on LabVIEW," International Journal of Smart Home, vol. 9, no. 6, pp. 1-10, 2015.

[7] L. Liang, M. Sun, S. Zhang, Y. Wen, and Y. W. Liu, "A integrate control system design of WPC with active T-foil and transom stern flap for vertical motion improvement," Journal of Computational Information Systems, vol. 9, 2015.

[8] M. Bernasconi, C. Choirat, and R. Seri, "The analytic hierarchy process and the theory of measurement," Management Science, vol. 56, no. 4, pp. 699-711, 2010.

[9] N. Bharadwaj, "Investigating the decision criteria used in electronic components procurement," Industrial Marketing Management, vol. 33, no. 4, pp. 317-323, 2004.

[10] C. Martínez-Olvera, "Entropy as an assessment tool of supply chain information sharing," European Journal of Operational Research, vol. 185, no. 1, pp. 405-417, 2008.

[11] Y. Zhang and B. E. Shenm, "Application of AHP in risk assessment for project and countermeasures for risk," Journal of Water Resources and Architectural Engineering, vol. 3, 2009.

[12] S. Sharmaa and N. Agrawalb, "Selection of a pull production control policy under different demand situations for a manufacturing system by AHP-algorithm," Computers and Operations Research, vol. 36, no. 5, pp. 1622-1632, 2009.

[13] V. Podvezko, "Application of AHP technique," Journal of Business Economics and Management, vol. 10, no. 2, pp. 181-189, 2009.

[14] L. C. Leung and D. Cao, "On consistency and ranking of alternatives in fuzzy AHP," European Journal of Operational Research, vol. 124, no. 1, pp. 102-113, 2000. 
[15] D. T. Wu and D. F. Li, "Shortcomings of analytical hierarchy process and the path to improve the method," Journal of Beijing Normal University (Natural Science), vol. 40, no. 2, pp. 264-268, 2004.

[16] J. Li and X. Wu, "Synthetic evaluation for urban rail transit line network planning scheme based on AHP-FUZZY method," Journal of Wuhan University of Technology, vol. 4, no. 2, pp. 205208, 2007.

[17] X. M. Wang, B. Zhao, and Q. L. Zhang, "Mining method choice based on AHP and fuzzy mathematics," Journal of Central South University: Science and Technology, vol. 39, no. 5, pp. 875-880, 2008.

[18] G. Xia, T. Luan, M. Sun et al., "Evaluation analysis for sortie generation of carrier aircrafts based on nonlinear fuzzy matterelement method," Journal of Intelligent \& Fuzzy Systems, vol. 31, no. 6, pp. 3055-3066, 2016.

[19] L. Liang, M. Sun, H. Shi, and T. Luan, "Design and analyze a new measuring lift device for fin stabilizers using stiffness matrix of euler-bernoulli beam," Plos one, vol. 12, no. 1, pp. 1-13, 2017.

[20] X. Guoqing, L. Tiantian, S. Mingxiao, and L. Yanwen, "Research on modeling of parallel closed-loop support process for carrier aircraft based on system dynamics," International Journal of Control and Automation, vol. 9, no. 11, pp. 259-270, 2016.

[21] X. Guo-Qing, L. Tian-Tian, S. Ming-Xiao, and L. Yan-Wen, "Dynamic analysis of catapult availability based on CBM," International Journal of Hybrid Information Technology, vol. 9, no. 10, pp. 31-42, 2016.

[22] S. Gao, X. Wu, G. Wang, J. Wang, and Z. Chai, "Fault diagnosis method on polyvinyl chloride polymerization process based on dynamic kernel principal component and fisher discriminant analysis method," Mathematical Problems in Engineering, vol. 2016, pp. 1-8, 2016.

[23] B. Jia, Y. Ma, X. Huang, Z. Lin, and Y. Sun, "A novel real-time ddos attack detection mechanism based on MDRA algorithm in big data," Mathematical Problems in Engineering, 2016.

[24] N. Han, Y. Song, and Z. Song, "Bayesian robust principal component analysis with structured sparse component," Computational Statistics and Data Analysis, vol. 109, pp. 144-158, 2017.

[25] P. G. Stevenson, N. K. Burns, S. D. Purcell et al., "Application of 2D-HPLC coupled with principal component analysis to study an industrial opiate processing stream," Talanta, vol. 166, pp. 119-125, 2017.

[26] S. Aouabdi, M. Taibi, S. Bouras, and N. Boutasseta, "Using multi-scale entropy and principal component analysis to monitor gears degradation via the motor current signature analysis," Mechanical Systems and Signal Processing, vol. 90, pp. 298-316, 2017. 


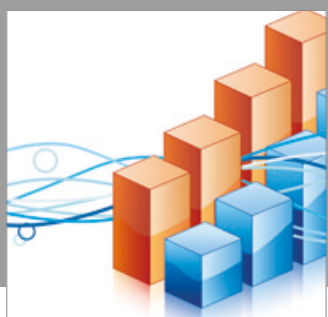

Advances in

Operations Research

vatersals

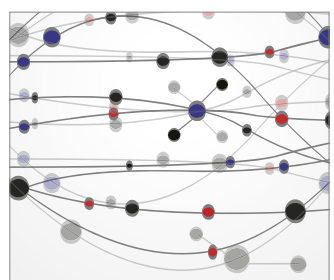

\section{The Scientific} World Journal
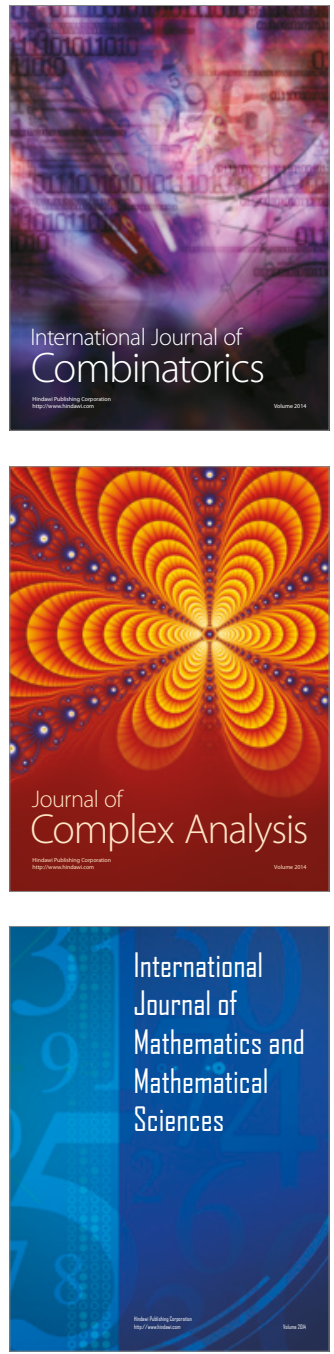
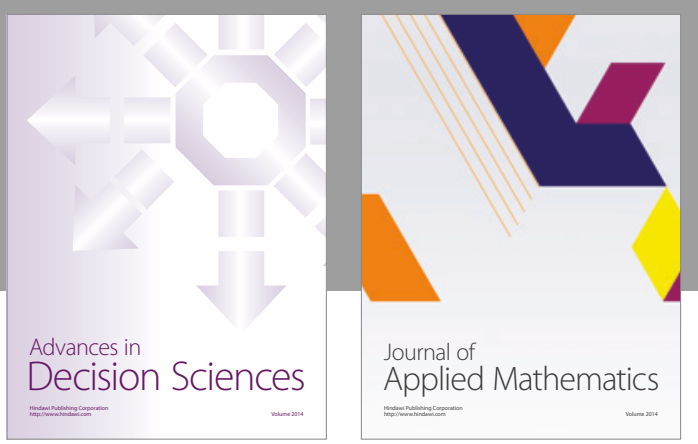

Algebra

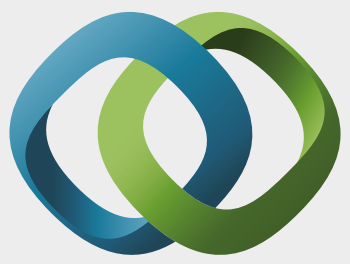

\section{Hindawi}

Submit your manuscripts at

https://www.hindawi.com
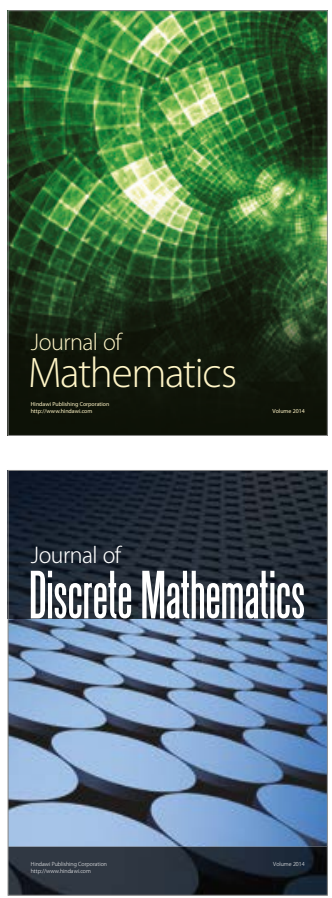

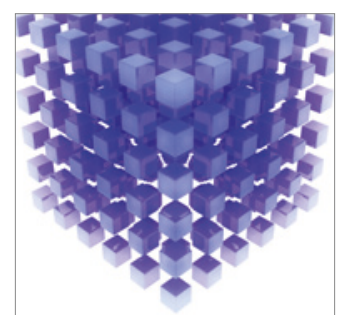

Mathematical Problems in Engineering
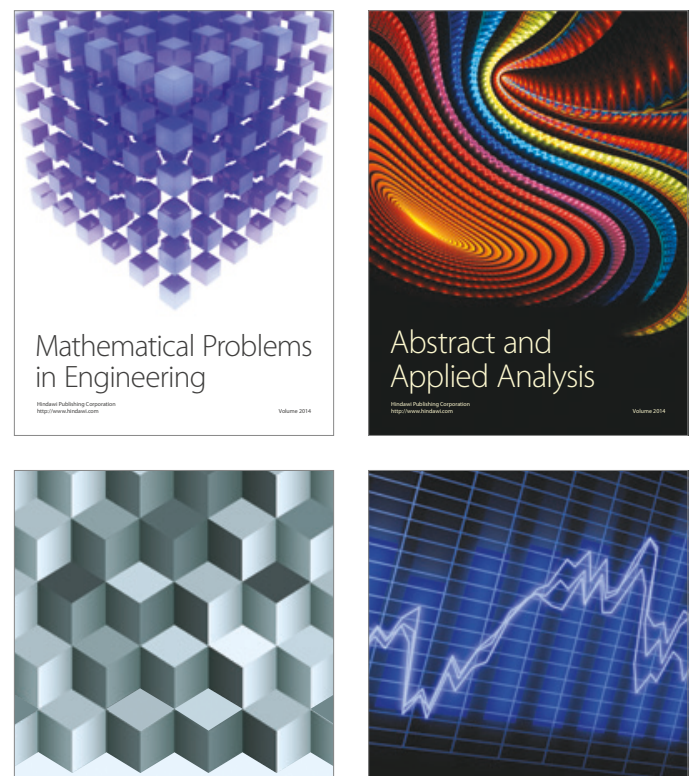

Journal of

Function Spaces

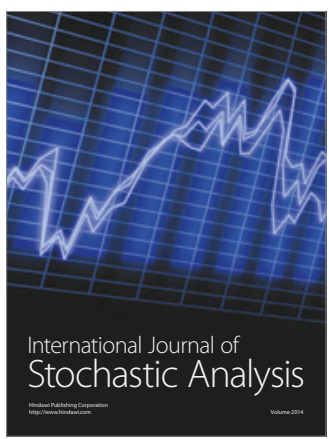

Probability and Statistics
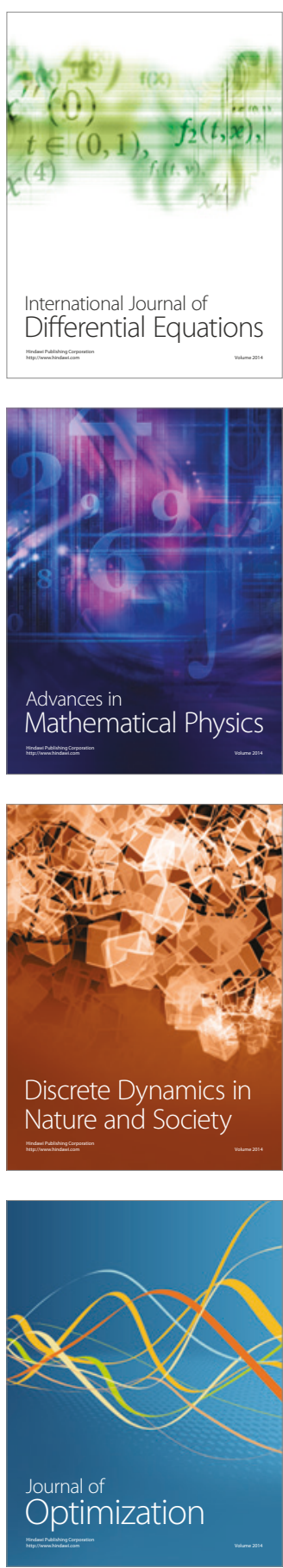